\title{
Supplementary material to "Stratospheric impact on the Northern Hemisphere winter and spring ozone interannual variability in the troposphere"
}

Junhua Liu' ${ }^{12}$, Jose M. Rodriguez ${ }^{2}$, Luke D. Oman ${ }^{2}$, Anne R., Douglass ${ }^{2}$, Mark A. Olsen ${ }^{3,}$, Lu Hu ${ }^{5}$

${ }^{1}$ Universities Space Research Association (USRA), GESTAR, Columbia, MD, USA

${ }^{2}$ NASA Goddard Space Flight Center, Greenbelt, MD, USA

${ }^{3}$ TriVector Services Inc., Huntsville, AL, USA

${ }^{4}$ NOAA/OAR/Office of Weather and Air Quality

${ }^{5}$ Department of Chemistry and Biochemistry, University of Montana, Missoula, MT, USA
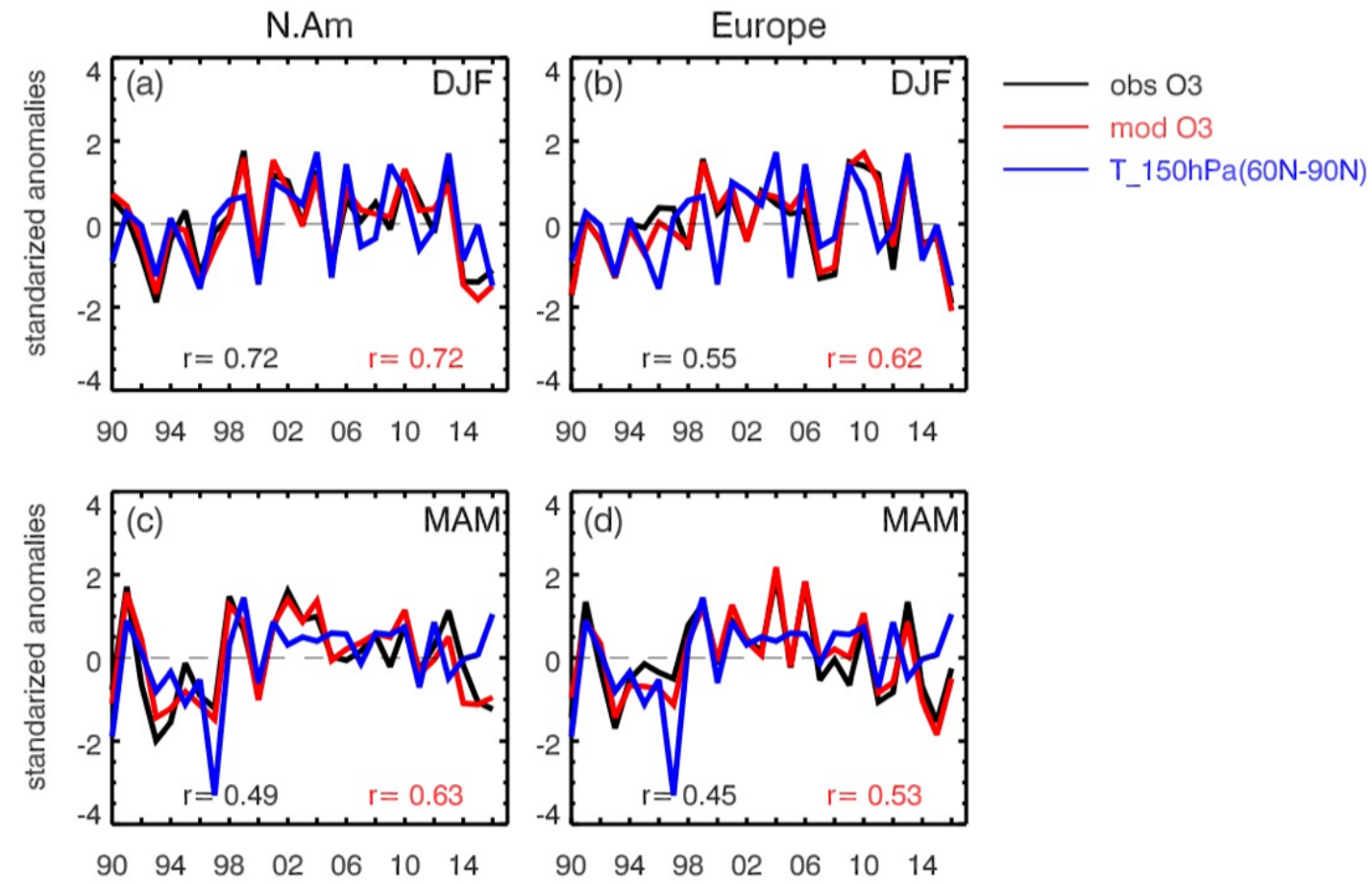

Figure S1: Standardized anomalies of observed (black) and simulated (red) $\mathrm{O}_{3}$ averaged over the North American stations (left) and over the European stations (right) at $200 \mathrm{hPa}$ with averaged temperature around the polar cap for latitudes north of $60^{\circ} \mathrm{N}$ (blue), which is a good measure of the overall temperature in the polar vortex. Correlation coefficient between observed $\mathrm{O}_{3}$ and temperature (black), simulated $\mathrm{O}_{3}$ and temperature (red) is shown in each panel. 

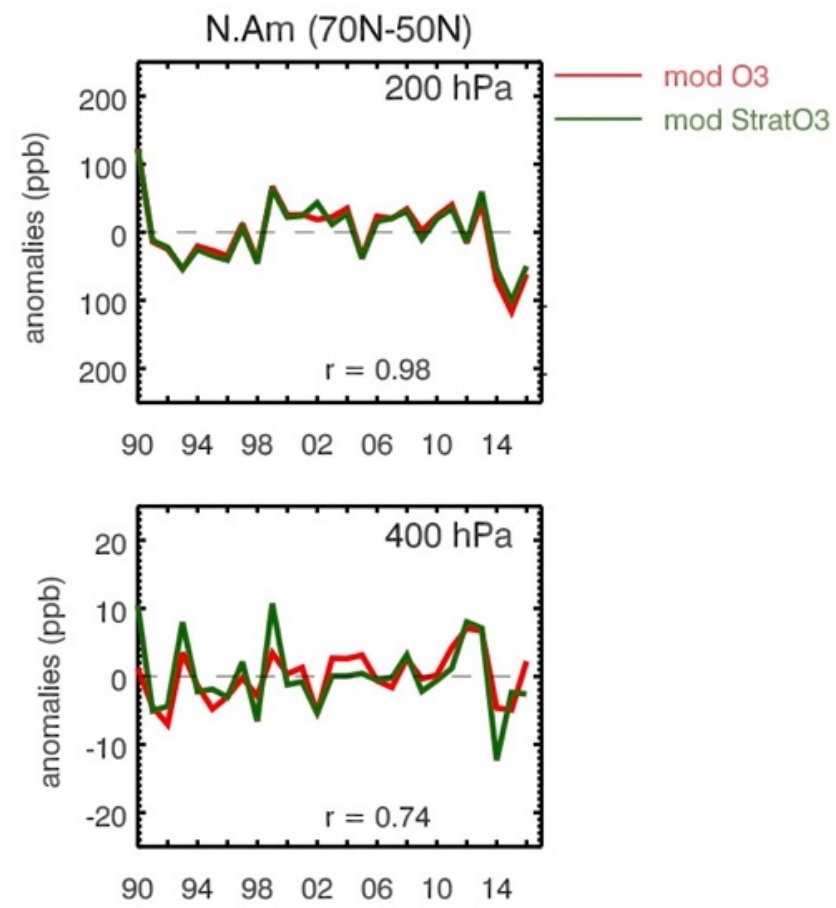

Figure S2: Time series plots of simulated ozone (red) and $\mathrm{StratO}_{3}$ (green) anomalies (unit: ppb) at $200 \mathrm{hPa}$ (top), $400 \mathrm{hPa}$ (bottom) averaged from the selected ozonesonde sites over the $50^{\circ} \mathrm{N}-7^{\circ} \mathrm{N}$ sub-region of North America in winter from 1990 to 2016.

N. America

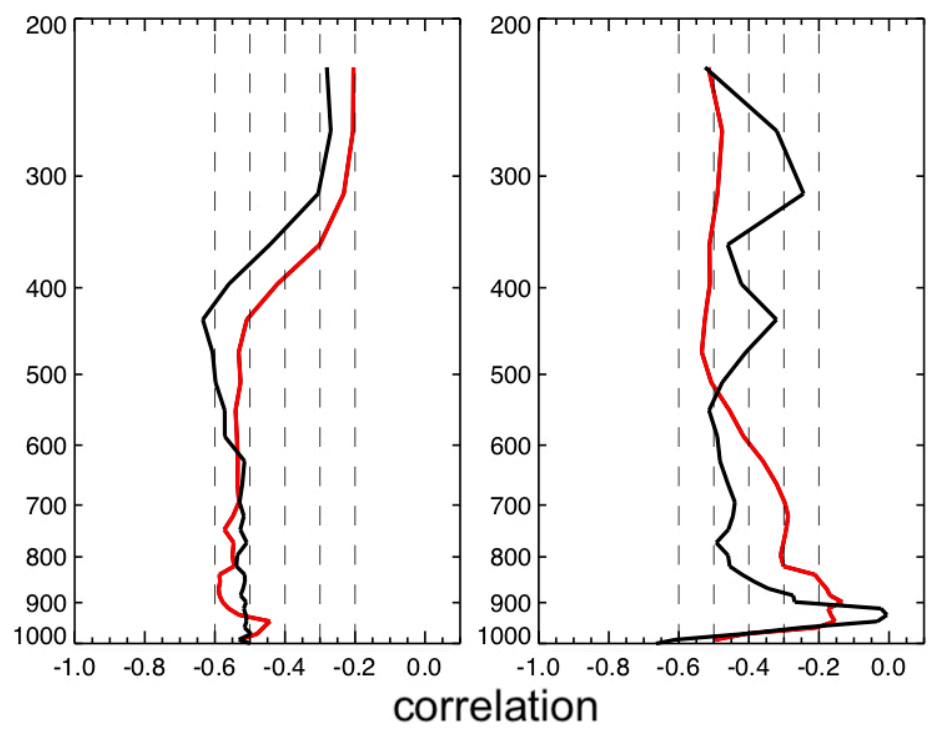

Europe

$r$ (obs-AO) $r(\bmod -A O)$

Figure S3: Profiles of correlations between $\mathrm{O}_{3}$ and $\mathrm{AO}$ index in winter from 1990 to 2016 averaged over the North American and the European stations. 